\title{
Le discariche dei rifiuti solidi urbani: evoluzione normativa, criteri di progettazione e tecnologie per il recupero e la valorizzazione del biogas
}

\author{
GIOSUE' PALOMBA ${ }^{1}$
}

${ }^{1}$ Affiliation not available

\begin{abstract}
La tematica approfondita riguarda i rifiuti solidi urbani, il loro smaltimento negli impianti predisposti, ovvero discariche controllate, la normativa afferente ed il recupero energetico. Tali tematiche rappresentano un problema molto importante per l'impatto che hanno sull'ecosistema. La problematica è dovuta agli elementi che si creano nell'impianto, come biogas e percolato, i quali devono essere controllati per evitare emissioni nell'ambiente circostante. Il seguente elaborato ha come obiettivo lo studio e l'analisi di un impianto per lo smaltimento dei rifiuti e le normative ad esso collegate, al fine di individuare il progresso delle già menzionate leggi negli anni e le procedure attuabili per la captazione del biogas. Lo studio prevede un percorso basato sulla ricerca e confronto di dati, partendo da un'analisi dei rifiuti che arrivano all'impianto fino ad arrivare alle tecnologie che lo rappresentano. Dopodiché verrà realizzato un modello virtuale con l'utilizzo di un software, ed un plastico in scala al fine di descriverlo al meglio. Mediante tali strumenti possiamo far emergere lo scopo dell'elaborato, ovvero quello di evidenziare come un corretto smaltimento dei rifiuti, nel rispetto delle norme, può portare ad una diminuzione delle problematiche legate alle discariche. Dal lavoro svolto, inoltre, si è individuata l'importanza delle tecnologie esistenti negli impianti, in grado di proteggere l'ambiente e riutilizzare i prodotti come nel caso del biogas.
\end{abstract}




\section{Introduzione}

La produzione dei rifiuti solidi urbani (RSU) e la loro gestione rappresentano degli aspetti fondamentali per lo studio inerente agli impianti di smaltimento. Definiamo innanzitutto cosa sia un rifiuto: secondo il decreto legislativo 152 del 2006, all'articolo 183 vi è la definizione generale attualmente vigente di rifiuto, ovvero qualsiasi sostanza od oggetto di cui il detentore si disfi o abbia l'intenzione o abbia l'obbligo di disfarsi. A questa poi vanno ad aggiungersi diverse specificazioni riguardo alla tipologia e allo stato fisico dello stesso infatti, un rifiuto, possiamo trovarlo allo stato solido, liquido e gassoso. In particolar modo, all'interno dello studio effettuato si trattano i rifiuti solidi urbani, che la normativa identifica come i rifiuti domestici, anche ingombranti, provenienti da locali e luoghi adibiti ad uso di civile abitazione. Lo studio dei rifiuti va incentrato su tre punti punti: produzione, classificazione e gestione. La produzione è un aspetto che ha fatto emergere, dallo studio effettuato, una correlazione con i parametri economici di una popolazione. Dal confronto dei dati dell'ISPRA ${ }^{1}$ è emerso che più c'è ricchezza, più c'è un aumento nella produzione dei rifiuti. Ma questa correlazione non cresce allo stesso modo, perché si è visto che le politiche di restrizione attuate sulla gestione dei rifiuti, hanno permesso un andamento di crescita inferiore agli aspetti economici ${ }^{2}$. La classificazione è invece un argomento molto importante e che ci fornisce le conoscenze adatte ad ottenere la differenziazione dei rifiuti e di conseguenza di ottimizzare la gestione. La normativa vigente utilizza per la classificazione il codice CER, che ci permette di ottenere le caratteristiche del rifiuto come provenienza, composizione, e pericolosità. Sull'ultimo aspetto, la pericolosità, bisogna porre molta attenzione, poiché occorrono impianti specifici per il trattamento di tali sostanze. La gestione è invece la fase operativa del ciclo dei rifiuti solidi urbani. Le fasi della gestione vanno dalla raccolta, per poi passare al trasporto, trattamento e smaltimento in discarica. La gestione risulta essere la parte nevralgica dell'intero ciclo dei rifiuti, poiché da essa ne derivano i quantitativi da smaltire. Come mostra la seguente figura, sono rappresentate le fasi della gestione, con annesse peculiarità ovvero tipi di raccolta esistenti, tipologia di mezze 
predisposti 1 trasporto, tipi di trattamenti e l'impianto per lo smaltimento. ${ }^{3}$

\section{Evoluzione normativa}

La normativa afferente ai rifiuti ed al loro ciclo risulta essere notevolmente ampia. Esistono normative sia a livello locale e sia a livello europeo, le quali pongono diversi obiettivi ma convergendo sullo scopo finale di una meticolosa classificazione, gestione e smaltimento dei rifiuti. Le normative vigenti europee e poi italiane, si fondano essenzialmente sul concetto di economica circolare. Tale principio tende a rendere la logica dell'economia autosufficiente ed ecosostenibile, in grado di reintegrare e rivalorizzare i materiali mediante un corretto susseguirsi dei meccanismi predisposti ${ }^{4,5,6,7,8,9,10,11,12,13,14,15,16}$.

La normativa europea vigente in materia di gestione dei rifiuti è il decreto del parlamento europeo del 30 maggio 2018 che va a modificare la direttiva europea del 18 novembre 2008. Con tali normative, l'unione europea, ha voluto fissare un quadro giuridico in merito al trattamento dei rifiuti nella comunità in modo tale da incentivare politiche di riciclaggio, riutilizzo e recupero dei rifiuti andando a ridurre in maniera considerevole i prodotti destinati allo smaltimento in discarica. Vengono confermati i principi nella gestione dei rifiuti e i diversi aspetti legati alla tutela ambientale.

La normativa Europea vigente sulle discariche è la direttiva del parlamento dell'UE 2018/850 del 30 maggio 2018 che va a modificare la direttiva 1999/31/CE del 26 aprile 1999. La 1999/31/CE è stata la normativa di base per le più recenti direttive, in quanto fissava rigidi requisiti operativi e tecnici per i rifiuti e le discariche, misure, procedure e orientamenti volti a prevenire o a ridurre il più possibile le ripercussioni negative sull'ambiente. Tra esse abbiamo in particolare l'inquinamento delle acque superficiali, delle acque freatiche, del suolo, dell'atmosfera, e sull'ambiente globale, compreso l'effetto serra, nonché i rischi per la salute umana risultanti dalle discariche di rifiuti, durante l'intero ciclo di vita. Con la modifica del 2018, la Comunità Europea 
ha voluto porre degli obiettivi futuri, con scadenza 2030-2035, agli stati membri. I punti introdotti vanno ad incentrare il discorso sull'evitare del tutto, o per la stragrande maggioranza, di portare rifiuti in discarica, con l'intenzione di riciclare e recuperare tutto ciò che è possibile $\mathrm{e}^{17,18,19,20,21,6,22}$

La normativa italiana sui rifiuti si basa sul decreto legislativo del 3 aprile $2006 \mathrm{n} .152$, rinominato "Norme in materia ambientale". Tale testo nasce in seguito ad una legge 308 introdotta nel 2004 che fissò dei requisiti in materia ambientale che furono, in seguito, racchiusi tutti nel Testo Unico del 2006. Il decreto pone anche un altro aspetto, precedentemente introdotto con la normativa europea, sulla gerarchia dei rifiuti, ed in particolare sui livelli di priorità, (Prevenzione, Preparazione al riutilizzo, Riciclaggio, Recupero, Smaltimento) i quali risultano essere i punti cardini per l'approccio alla gestione dei rifiuti. in materia di rifiuti, vengono confermati gli aspetti legati alle responsabilità economiche di chi inquina paga, della responsabilità condivisa sulla produzione, il principio della prossimità e dell'autosufficienza.

La normativa italiana sugli impianti di smaltimento attualmente vigente è il decreto legislativo n.121 del 3 settembre 2020 , che va a modificare ed integrare il vecchio d.lgs 36/2003. Il decreto appena emanato va ad attuare le modifiche introdotte con la normativa europea sulle discariche, ovvero la 2018/850 del 30 maggio 2018. nel decreto sono contenuti tutti gli aspetti inerenti alla costruzione, ubicazione e classificazione degli impianti, nonché alla tipologia di rifiuti ammessi in tali impianti. Sono state irrigidite le regole degli impianti e sulla classificazione degli stessi.

\section{Discarica controllata}

La discarica controllata costituisce l'unico impianto riconosciuto dalla normativa per lo smaltimento dei rifiuti. Tali impianti sono alimentati dagli scarti ottenuti dai trattamenti dei rifiuti quali ceneri, oppure FOS (frazione organica stabilizzata), e da rifiuti dai quali non è stato possibil effettuare un recupero energetico o di materia. Gli impianti di smaltimento di rifiuti solidi urbani 
prevedono generalmente:

- Un'area di ingresso ben distinta

- Un'area per la pesa e accettazione dei rifiuti

- Un' area per l'imballaggio

- Un bacino (nel caso di trincea) per depositare i rifiuti

- Un'area pianeggiante (nel caso di sistemi sopraelevati) per depositare i rifiuti

- Una rete di raccolta delle acque meteoriche

- Delle barriere per isolare i rifiuti dall'ambiente

- Un impianto per il trattamento del percolato

- Pozzi di estrazione del biogas

- Impianto pompaggio e trasformazione biogas

La progettazione delle aree deve prevedere il rispetto dei criteri contenuti negli allegati della normativa sulle discariche. In riferimento al caso studio dal quale prende forma il seguente elaborato, occorre specificare che al fine di evitare che l'impianto risulti essere fonte di inquinamento per l'ecosistema, devono essere previste barriere adeguate per evitare la fuoriuscita di biogas e percolato dalla discarica, e questi prodotti devono essere prelevati perché possibile fonte di pericolo per l'uomo. Il percolato viene drenato mediante un apposito impianto per poi essere trattato in un impianto specifico. Mentre per quanto riguarda il biogas, data la sua natura di gas infiammabile, viene pompato dalla discarica mediante uno specifico impianto, filtrato e viene successivamente utilizzato come combustibile per la produzione di energia elettrica e termica. Il prelievo dal sito avviene mediante pozzi di estrazione, generalmente verticali, poi collegati alla rete di regolazione e pompaggio. Gli impianti per la combustione si compongono di diverse parti, trale più importanti abbiamo il motore endotermico o una caldaia, per il recupero energetico, è una torcia o camera di combustione, utilizzata in caso di guasto e/o quantità eccessive di biogas, poiche quest'ultimo non essendo stoccabile, deve essere distrutto. La norma fissa anche questo caso i criteri. 
La fase operativa e gestionale negli impianti risulta molto importante perché bisogna sottolineare che devono essere garantite accuratamente le condizioni di sicurezza per l'uomo. bisogna andare a controllare eventuali fughe incontrollate di gas, guasti e cercare di ottenere sempre una produzione opportuna e commisurata all'impianto.

In fine, per rendere meno di impiatto la struttura, vengono previsti delle coperture vegetative del corpo deposizionale e una vegetazione arborea a fusto alto perimetralmente al sito.

\section{Progettazione}

La progettazione è consistita nell'andare a realizzare un modello virtuale in 3D di un impianto.

La progettazione è iniziata partendo dai dati dell'impianto, effettuando delle stime di produzione in funzione della grandezza. Le stime sono frutto di un comparazione di dati di impianti analoghi.

- Volume corpo deposizionale: circa $1.700 .00 \mathrm{~m}^{3}$

- Quantità stimata di RSU conferita annualmente: $100.000 \mathrm{~m}^{3}$

- Quantità stimata di Biogas ottenibile: circa $1000 \mathrm{~m}^{3} / \mathrm{h}$

- Energia ottenibile: $4500 \mathrm{KW} / \mathrm{h}$ di cui $1300 \mathrm{WK} / \mathrm{h}$ di energia elettrica

Lo step successivo è stata una modellazione prima in due dimensioni e poi n $3 \mathrm{D}$ partendo da AutoCAD ${ }^{\circledR}$. Con l'ausilio di ArchiCAD® e di Twinmotion ${ }^{\circledR}$ si è realizzato un modello 3D ed è stato renderizzato, ottenendo così un risultato molto realistico.

L'impianto previsto si compone di:

- Area accettazione e pesa rifiuti

- Edificio uffici, servizi

- Magazzino attrezzature con area per imballaggio rifiuti

- 2 stazioni di regolazione 
- 20 pozzi di estrazione verticale

- Bacino per i rifiuti adeguatamente drenato ed isolato

- Centrale di pompaggio

- Impianto di filtraggio gas

- Torcia di combustione

- 2 Motori endotermici

- Trasformatore elettrico

- Cabina elettrica

- Impianto per il trattamento del percolato

- Vasca di prima pioggia

- Impianto lavaggio camion

- Strada perimetrale con cunetta laterale per raccolta acque meteoriche

La progettazione si è conclusa con foto e video render dell'impianto, e la realizzazione di un plastico.

\section{Conclusioni}

Dallo studio dell'elaborato di tesi da cui devira il seguente articolo, sono emersi due punti interessanti. Il primo riguarda la produzione e gestione dei rifiuti, in quanto mediante il progresso normativo, si vuole portare la gestione all'ottimizzazione massima, senza quindi portare i rifiuti in discarica. Il riferimento a questa osservazione nasce il secondo punto di riflessione sull'utilità delle discariche. Si può affermare che tali impianti risultano essere ancora validi e che se predisposti in un certo modo, possono garantire un efficiente recupero energetico. Si possono ritenere validi questi impianti, perché rappresentano il segmento utile nella fase di transizione prevista per il raggiungimento dell'obbiettivo posto dalla normativa di azzerare il conferimento. Questo perché tale 
obbiettivo non è immediato, e c'è bisogno di un corretto smaltimento, tanto più se anche da esso si riesce a recupere energia. Bisogna sottolineare, inoltre, che anche da un impianto di discarica è possibile ottenere un recupero energetico e che, nonostante lo studio si sia incentrato su una nuova costruzione, le politiche di un efficientamento e di recupero energetico dalle discariche valgono anche per impianti già esistenti, con la possibilità di andare ad effettuare bonifiche.

\section{References}

1.Rapporto Rifiuti Urbani - Edizione 2019.

2.Optimization of Field Inspection Method for Odour Impact Assessment. chemical engineering 23, (2011).

3.ISPRA Catasto Nazionale Rifiuti.

4.Performance study of e-nose measurement chamber for environmental odour monitoring. Chemical Engineering 30 30, (2012).

5.A comparative and Critical Evaluation of Different Sampling Materials in the Measurement of Odour Concentration by Dynamic Olfactometry. CHEMICAL ENGINEERING TRANSACTIONS 30 30, (2012).

6.Naddeo, V., Belgiorno, V. \& Zarra, T. Procedures for Odour Impact Assessment. in Odour Impact Assessment Handbook 187-203 (John Wiley \& Sons Inc., 2012). doi:10.1002/9781118481264.ch7. 
7.Zarra, T., Galang, M. G., Ballesteros, F., Belgiorno, V. \& Naddeo, V. Environmental odour management by artificial neural network - A review. Environment International 133, 105189 (2019).

8.A novel tool for estimating the odour emissions of composting plants in air pollution management. Issue 4 11, 477-486 (2013).

9.Scannapieco, D., Naddeo, V. \& Belgiorno, V. Sustainable power plants: A support tool for the analysis of alternatives. Land Use Policy 36, 478-484 (2014).

10.Naddeo, V., Belgiorno, V., Zarra, T. \& Scannapieco, D. Dynamic and embedded evaluation procedure for strategic environmental assessment. Land Use Policy 31, 605-612 (2013).

11.Murena, A. et al.. Water-Energy Nexus: Evaluation of the Environmental Impact on the National and International Scenarios. in Frontiers in Water-Energy-Nexus-Nature-Based Solutions Advanced Technologies and Best Practices for Environmental Sustainability 33-35 (Springer International Publishing, 2019). doi:10.1007/978-3-030-13068-8.

12.Frontiers in Water-Energy-Nexus-Nature-Based Solutions Advanced Technologies and Best Practices for Environmental Sustainability. (Springer International Publishing, 2020). doi:10.1007/978-3-030-13068-8.

13.Hasan, S. W., Liu, H., Naddeo, V., Puig, S. \& Yip, N. Y. Editorial: Environmental technologies for the sustainable development of the water and energy sectors. Water Science and Technology 81, iii-iv (2020).

14.Environmental Technologies for the Sustainable Development of the Water and Energy Sectors. (IWA Publishing, 2020). doi:10.2166/9781789062328.

15.Naddeo, V. \& Korshin, G. Water energy and waste: The great European deal for the environment. Science of The Total Environment 764, 142911 (2021). 
16.Naddeo, V. \& Taherzadeh, M. J. Biomass valorization and bioenergy in the blue circular economy. Biomass and Bioenergy 149, 106069 (2021).

17.Nesticò, A., Elia, C. \& Naddeo, V. Sustainability of urban regeneration projects: Novel selection model based on analytic network process and zero-one goal programming. Land Use Policy 99, 104831 (2020).

18.Lado, J. J. et al.. Performance analysis of a capacitive deionization stack for brackish water desalination. Desalination 501, 114912 (2021).

19.Zarra, T., Galang, M. G. K., Ballesteros, F. C., Belgiorno, V. \& Naddeo, V. Instrumental Odour Monitoring System Classification Performance Optimization by Analysis of Different PatternRecognition and Feature Extraction Techniques. Sensors 21, 114 (2020).

20.Oliva, G. et al.. Next-generation of instrumental odour monitoring system (IOMS) for the gaseous emissions control in complex industrial plants. Chemosphere 271, 129768 (2021).

21.Senatore, V. et al.. Innovative membrane photobioreactor for sustainable CO2 capture and utilization. Chemosphere 273, 129682 (2021).

22.Zarra, T., Giuliani, S., Naddeo, V. \& Belgiorno, V. Control of odour emission in wastewater treatment plants by direct and undirected measurement of odour emission capacity. Water Science and Technology 66, 1627-1633 (2012).

23.Europeo, P. Direttiva 2018/850.

24.DIRETTIVA (UE) 2018/851.

25.DECRETO LEGISLATIVO 3 aprile 2006, n. 152.

26.DECRETO LEGISLATIVO 3 settembre 2020, n. 121. 
Figure Captions

Figure 1. Schema gestione dei RSU

Figure 2. Foto render impianto

Figure 3. Foto rendering impianto 


\section{Figures}

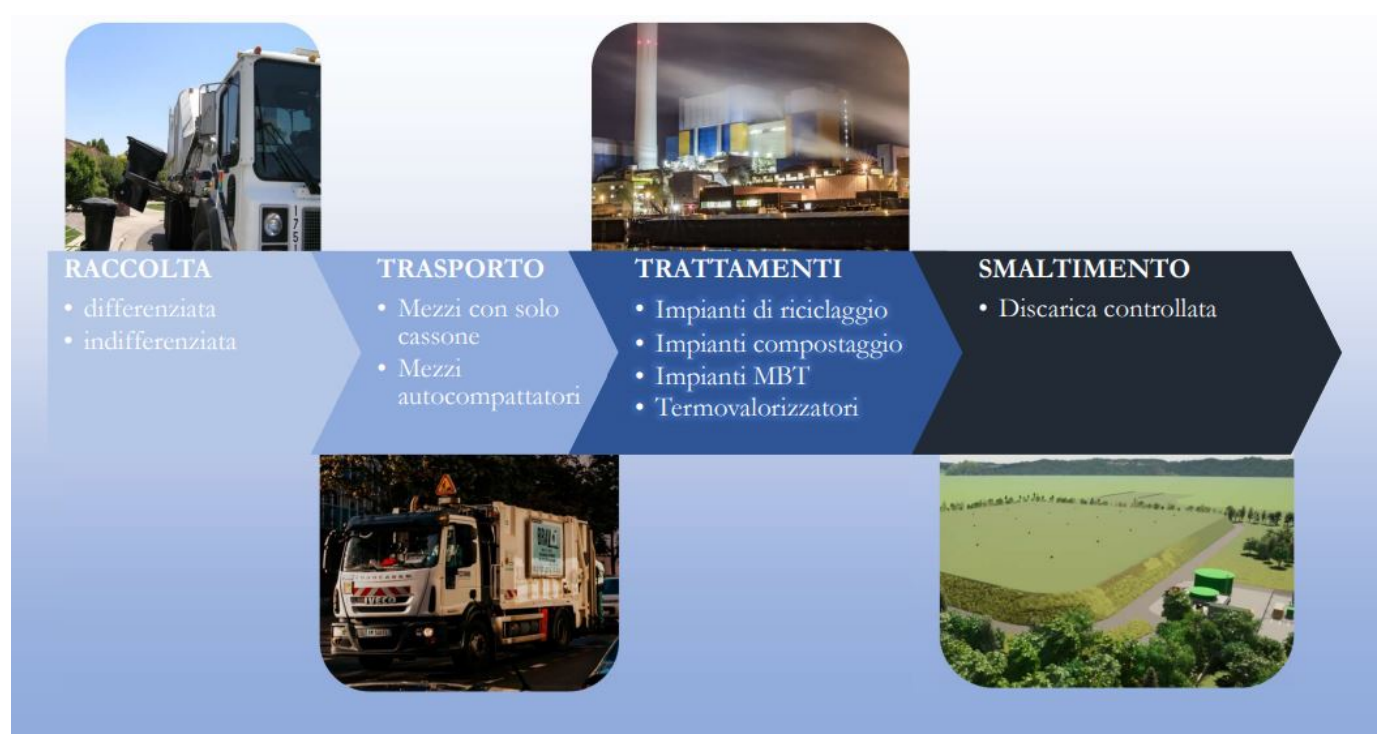

Figure 1: Schema gestione dei RSU

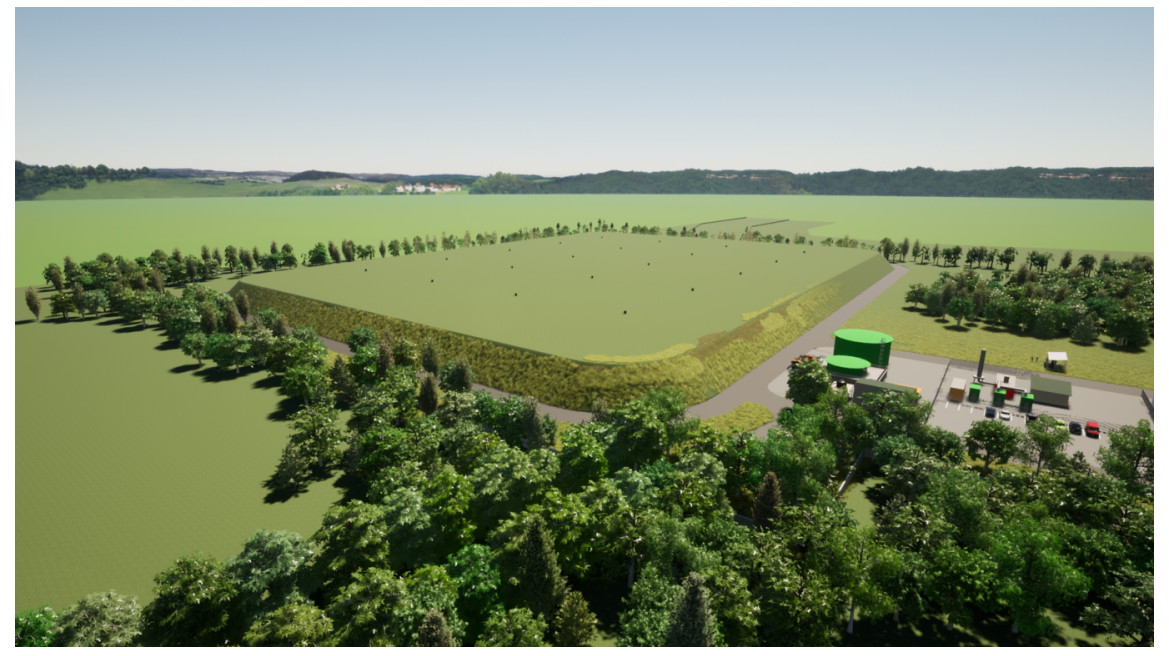

Figure 2: Foto render impianto 


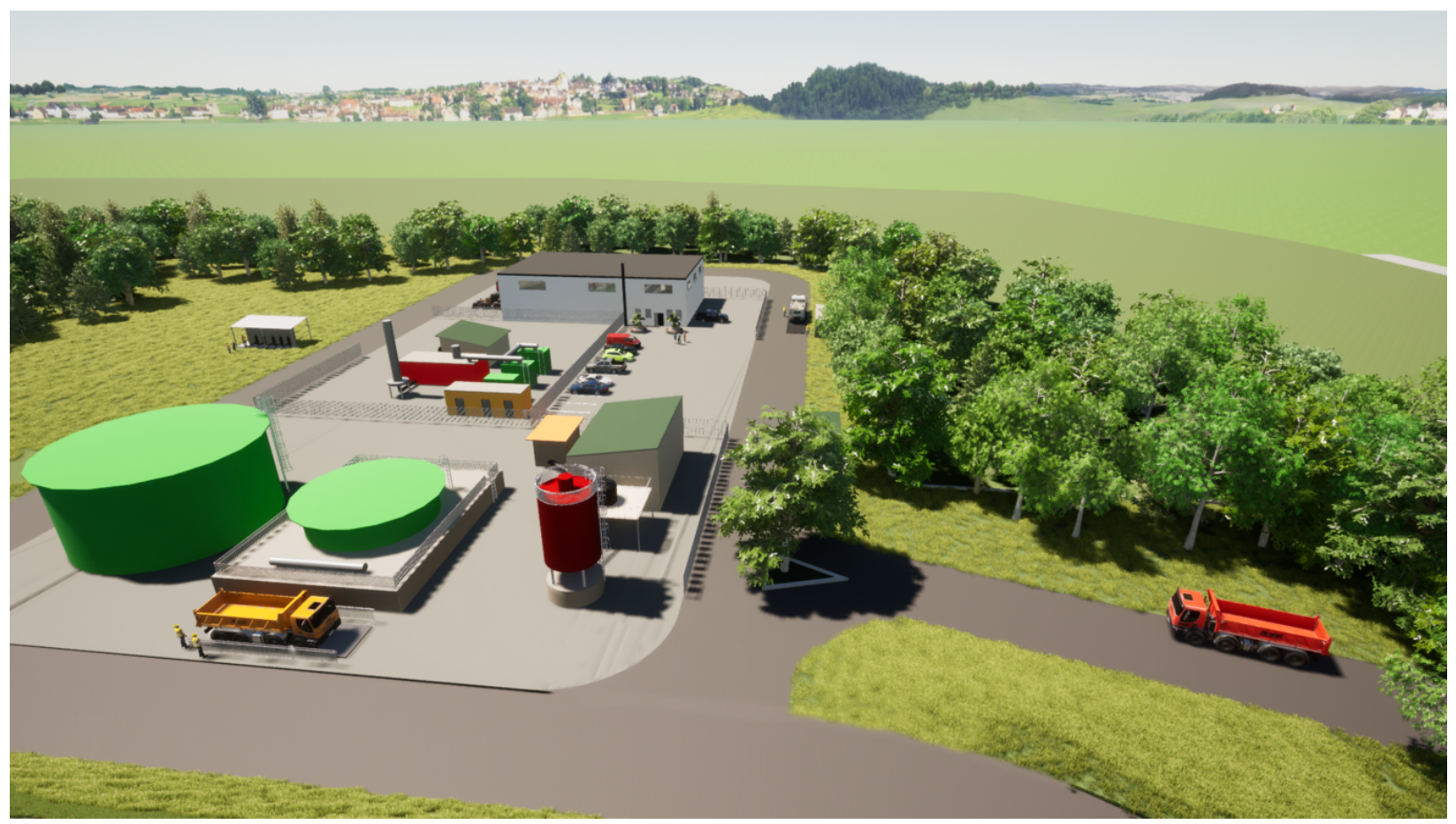

Figure 3: Foto rendering impianto 